\title{
DISCRETIZATION ERROR DOMINANCE OVER SUBGRID TERMS IN LARGE EDDY SIMULATION OF COMPRESSIBLE SHEAR LAYERS IN 2D
}

\author{
BERT VREMAN, BERNARD GEURTS AND HANS KUERTEN \\ Department of Applied Mathematics, University of Twente, P.O. Box 217, 7500 AE Enschede, The Netherlands
}

SUMMARY

Second-and fourth-order-accurate spatial discretization methods give rise to discretization errors which are larger than the corresponding subgrid terms in large eddy simulation of compressible shear layers in $2 \mathrm{D}$, if the ratio between the filter width and the grid spacing is close to one. Even if an exact representation for the subgrid-scale contributions is assumed, large eddy simulation is accurate only if this ratio is sufficiently larger than one. In that regime fourth-order methods are more accurate than second-order methods. An analysis of the data obtained from two-dimensional direct numerical simulations of compressible shear layers substantiates these assertions.

At sufficiently high Reynolds number the occurrence of small-scale structures in turbulent flows prevents a direct numerical simulation (DNS) of the Navier-Stokes equations, even in simple geometries. Therefore much attention is paid to large eddy simulation (LES), in which only the large scales are solved time-accurately, while the small scales are modelled. In order to obtain the LES formulation, the Navier-Stokes equations are filtered. As a result, two types of terms occur: terms expressed in the filtered variables and the so-called subgrid terms. The first class of terms is approximated by a discretization method, which introduces discretization errors, whereas the second class of terms is represented by a subgrid model and subsequently discretized. Much research effort has been and is still put into the development of good subgrid models. However, it seems reasonable to employ a subgrid model only if the discretization errors are small compared to the subgrid terms. ${ }^{1}$ The magnitude of the subgrid terms has widely been discussed in literature; estimates and scalings are presented in References 2-4, while the terms are explicitly calculated in, for example, Reference 5. It is also argued in Reference 1 that the ratio between filter width $(\Delta)$ and grid spacing $(h)$ of the coarse grid used for LES influences the accuracy. In practice LES is usually performed for $\Delta=h$ (e.g. References 6 and 7) or $\Delta=2 h$ (e.g. Reference 8). In References 9 and 10 several values for the ratio $\Delta / h$ are considered and it is found that the total simulation error (arising from the modelling and the discretization) for schemes that are second-order-accurate in space, is $\Delta=2 h$.

In this paper we use DNS results in order to calculate not only the magnitude of the subgrid terms but also the magnitude of the discretization errors. It will be shown that for both secondand fourth-order-accurate spatial discretizations, the discretization errors dominate the subgrid terms, in case $\Delta=h$. Only if $\Delta$ is sufficiently larger than $h$, fourth-order-accurate methods yield considerably smaller discretization errors than second-order-accurate methods.

CCC 0748-8025/94/100785-06

(c) 1994 by John Wiley \& Sons, Ltd.

Received 5 July 1993 Revised 20 October 1993 
To obtain these results, we will analyse in detail a DNS of the temporal mixing layer. Similar conclusions can be drawn from a study of the flow over a flat plate. As in References 11 and 12 we will only address the two-dimensional problem in order to demonstrate the numerical procedure which is used for analyzing the DNS data. The same procedure can be applied to more complicated and physically more realistic flows in three dimensions. Results of threedimensional studies will be presented elsewhere.

In the following we will first describe the simulation and show that the DNS is accurate by comparing the results obtained from increasingly fine grids $\left(64^{2}, 128^{2}\right.$ and $256^{2}$ cells, respectively). Then, we will filter the data and compare the discretization errors with the subgrid terms, calculated on an LES grid $\left(64^{2}\right.$ cells). Moreover, we will compare the results obtained from some large eddy simulations at different $\Delta / h$ ratios (with $\Delta$ held fixed) with the filtered DNS results.

The compressible temporal mixing layer is treated on a uniform orthogonal grid. Periodic boundary conditions are imposed in the $x_{1}$-direction, and in the $x_{2}$-direction the boundaries are treated as free slip walls. The basic initial profile is the hyperbolic tangent profile. A low amplitude perturbation consisting of a sinusoidal wave superimposed on uniform noise and multiplied with a Gaussian in the $x_{2}$-direction is added to the basic initial profile. This sinusoidal wave has a wavelength corresponding to the most unstable mode as predicted by linear stability theory. ${ }^{13}$ We put the length of the domain $(L)$ equal to twice the wavelength of the most unstable mode. The Reynolds number, based on the free stream velocity and half the initial vorticity thickness, is equal to 200 . The convective Mach numbers ${ }^{14}$ is chosen to be $0 \cdot 2$, implying that compressibility effects are small. As a numerical method we use a finite volume approach with central spatial differencing which is second-order-accurate in space, ${ }^{15}$ and a second-order-accurate explicit four-stage Runge-Kutta method for the time integration.

The results of the simulations show that with this numerical method an accurate DNS is obtained. The simulations are qualitatively correct; the fundamental instability grows, saturates and produces two vortices, which move towards each other and merge, as reported in Reference 16. The DNS is also quantitatively correct, if the resolution is adequate. This is supported by Figure 1, which presents the vorticity thickness, as defined in Reference 16, obtained from simulations with $64^{2}, 128^{2}$ and $256^{2}$ cells, respectively. We conclude that a DNS with $256^{2}$ cells provides accurate data which can be used to investigate the role of discretization errors and subgrid terms in LES studies.

In the following, a representative subgrid term and the corresponding discretization error are compared for two different spatial discretizations and several filter widths. A filter operation extracts the large scale part $\bar{f}$ from a quantity $f$. Here the top-hat filter ${ }^{1}$ with filter width $\Delta$ is employed. For compressible flow, a related filter operation is commonly used, ${ }^{8}$ namely $\tilde{f}=\overline{\rho f} \mid \bar{\rho}$, where $\rho$ is the density.

The LES formulation is obtained by applying the 'bar-filter' to the Navier-Stokes equations. The filtered continuity equation does not give rise to a subgrid term, if we consider the filtered velocity $\tilde{u}_{j}$ instead of $\bar{u}_{j}$. In the filtered momentum equation subgrid terms do appear:

$$
\partial_{t} \overline{\rho u_{i}}+\partial_{j} \overline{\rho u_{i} u_{j}}+\partial_{i} \bar{p}-\partial_{j} \bar{\sigma}_{i j}=0
$$

Here the symbols $\partial_{t}$ and $\partial_{j}$ are abbreviations of the partial differential operators $\partial / \partial t$ and $\partial / \partial x_{j}$, respectively. Moreover, the summation convention for repeated indices is used. The time is denoted by $t$, while $p$ is the pressure and $\sigma_{i j}$ is the viscous stress tensor for a Newtonian fluid.

In the following, we focus on the non-dissipative part of the filtered momentum equation 


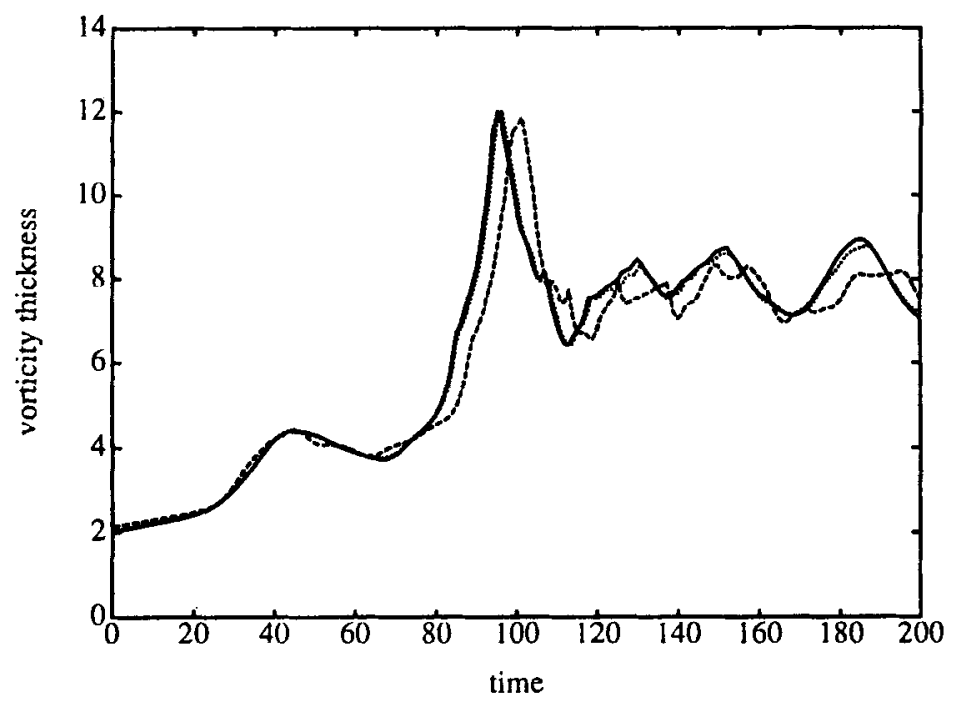

Figure 1. Vorticity thickness for simulations with a $64^{2}$-grid (dashed), a $128^{2}$-grid (dotted) and a $255^{2}$-grid (solid)

in the $x_{1}$-direction, which contains a representative convective term and a pressure term. Within an LES the first term, $\partial_{j} \overline{\rho u_{1} u_{j}}$, cannot be calculated directly, since it is not expressed in terms of the filtered variables. Consequently, one writes

$$
\partial_{j} \overline{\rho u_{1} \bar{u}_{j}}=\partial_{j}\left(\bar{\rho} \tilde{u}_{1} \tilde{u}_{j}\right)+\alpha
$$

where $\alpha$ is the subgrid term. However, the first term on the right-hand side of this equation cannot be calculated exactly either, since the quantities $\bar{\rho}$ and $\tilde{u}_{j}$ are only known at discrete grid points. Similarly, the pressure term introduces a discretization error. Therefore, denoting the discrete partial derivative operator by $\delta_{j}$, we obtain

$$
\partial_{j}\left(\bar{\rho} \tilde{u}_{1} \tilde{u}_{j}\right)+\partial_{1} \bar{p}=\delta_{j}\left(\bar{\rho} \tilde{u}_{1} \tilde{u}_{j}\right)+\delta_{1} \bar{p}+\beta
$$

in which $\beta$ is the discretization error. So, whereas formally $\partial_{j} \bar{\rho} u_{1} \bar{u}_{j}+\partial_{1} \bar{p}$ is required, only $\delta_{j}\left(\bar{\rho} \tilde{u}_{1} \tilde{u}_{j}\right)+\delta_{1} \bar{p}$ is available in an LES study. Consequently, there is a total discrepancy $\alpha+\beta$, since

$$
\partial_{j} \overline{\rho u_{1} u_{j}}+\partial_{1} \bar{p}=\delta_{j}\left(\bar{\rho} \tilde{u}_{1} \tilde{u}_{j}\right)+\delta_{1} \bar{p}+\alpha+\beta
$$

Whereas the subgrid term is usually modelled with a subgrid model, the discretization error $\beta$ is not taken into account. The main question we address is whether this is justified.

Next we turn to the calculation of $\alpha$ and $\beta$. The determination of $\alpha$ and $\beta$ proceeds in a few steps. First the $256^{2}$ DNS data are filtered with a filter width that corresponds with at least four cells in each direction. As a result, the filter operation yields $\bar{\rho}, \bar{p}, \tilde{u}_{i}$ and $\bar{\rho} \bar{u}_{i} u_{j}$ defined on the $256^{2}$ grid. Since after the filtering these quantities are relatively smooth, $\alpha$, defined by equation (1), is accurately calculated with the fourth-order five-point central difference on the $256^{2}$ grid (the relative error is less than 0.1 per cent). Finally, $\alpha$ is restricted to a coarse grid, which contains $64^{2}$ cells of size $h$. This LES grid is considerably coarser than the DNS grid, but should be sufficiently fine to perform a well resolved LES. Since the operator $\delta_{j}$ in equation 
(2) is a discretization of the partial derivative on the coarse LES grid, the discretization error $\beta$ is readily calculated as the difference between the fine and coarse grid derivatives.

Results will be shown for two operators $\delta_{j}$ : the above-mentioned second-order finite-volume discretization and the fourth-order five-point central difference. As a typical example, the DNS results at $t=80$ are used to calculate the magnitudes of $\alpha$ and $\beta$. Figure 2 presents $\|\alpha\|,\|\beta\|$ and $\|\alpha+\beta\|$ for the second- and fourth-order method. Here $\|\cdot\|$ denotes the discrete $\mathrm{L}_{2}$-norm applied to quantities defined on $64^{2}$ cells. The variable $r$ is the ratio $\Delta / h$, in which the coarse grid size $h$ is kept constant. We observe that $\alpha \sim \Delta^{2}$ to close approximation and we have checked that the components of the turbulent stress tensor $\left(\tau_{i j}=\bar{u}_{i} u_{j}-\tilde{u}_{i} \tilde{u}_{j}\right)$ are of order $\Delta^{2}$ as well. Next, we compare the second-order with the fourth-order method. If $\Delta=h$, the discretization error $\beta$ is larger than the subgrid term $\alpha$ for both methods. Consequently, in this case it is not useful to perform LES even with a perfect subgrid model, since a large discretization error will disturb the simulation, as shown below. If $\Delta$ is sufficiently larger than $h$, which corresponds to smoother fields on the same $64^{2}$ grid, one finds that $\alpha$ is considerably larger than $\beta$. In this regime the second-order method shows a small decrease of the discretization error for increasing $\Delta$, whereas the fourth-order finite difference method shows a rapidly decaying discretization error for increasing $\Delta$. Hence, in this regime the fourth-order method is considerably more accurate than the second-order method.

In order to show the effects of the discretization errors in an actual LES we compare the vorticity thickness of the filtered DNS with some corresponding large eddy simulations for different ratios $\Delta / h$ with $\Delta=L / 32$ held fixed (Figure 3). We use the Smagorinsky model for the subgrid terms ${ }^{1}$ and employ the second-order spatial discretization. A very low value of the Smagorinsky constant $C_{\mathrm{S}}$ was used until the initial perturbations have saturated $(t \approx 40)$, in order to prevent excessive dissipation in the laminar regime. ${ }^{17}$ After this transient stage we use the typical value $C_{\mathrm{S}}=0 \cdot 2 .{ }^{1}$ When we consider the vorticity thickness of the three large eddy simulations, we observe that the discretization error strongly influences the quantitative

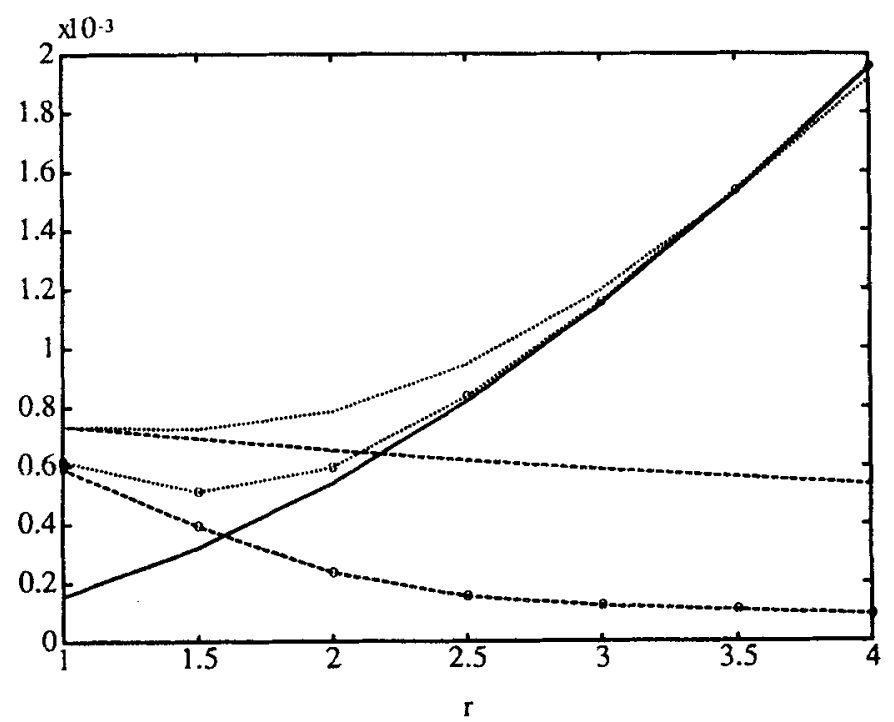

Figure 2. $\|\alpha\|$ (solid), $\|\beta\|$ (dashed) and $\|\alpha+\beta\|$ (dotted), obtained with a second-order (no marker) and a fourthorder-accurate method (marker ' $O$ ') 


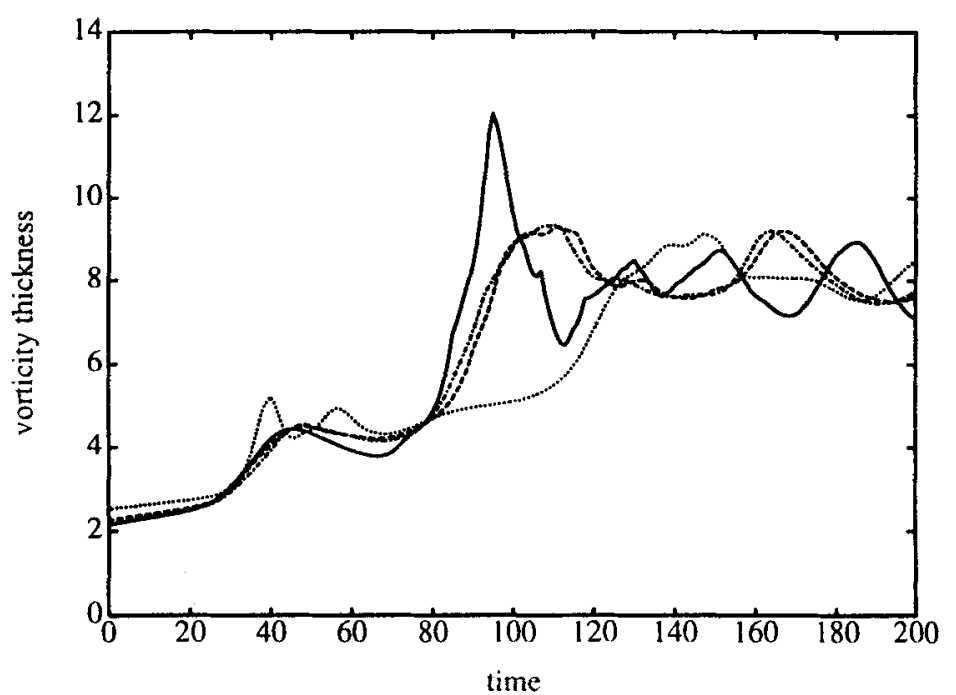

Figure 3. Vorticity thickness for a filtered DNS (solid) and the corresponding large eddy simulations with $\Delta=h$ (dotted), $\Delta=2 h$ (dashed) and $\Delta=4 h$ (dashed-dotted)

predictions of the simulation with $\Delta=h$. However, even at $\Delta=4 h$, in which case the discretization error is small, the discrepancy with the filtered DNS is quite large, due to the poor subgrid modelling. We even observed, by varying the constant $C_{S}$, that the LES predictions based on Smagorinsky's model are not as accurate as those obtained with an underresolved DNS on the same grid. ${ }^{17}$ The subgrid modelling for this two-dimensional problem might be improved when, for example, a stochastic backscatter is added to Smagorinsky's model. ${ }^{11}$

As a conclusion, for the compressible mixing layer in two dimensions we have shown that the discretization errors are larger than the subgrid terms if the filter width $\Delta$ equals the grid size $h$, although most modern LES research is based on the proposition $\Delta=h$. This was observed for both a second-order- and a fourth-order-accurate discretization method. Consequently, the accuracy of LES is not adequate in this regime and the quantitative predictions are questionable. Furthermore, we have presented numerical evidence to support the hypothesis that simulations with $\Delta$ sufficiently larger than $h$ result in smaller numerical errors. Finally, we observed that this increased accuracy is only signifficant if higher than second-order-accurate discretizations are used. To find out whether these results can be generalized, other types of flows, different filters and 3D extensions should be considered. With respect to the first issue, similar conclusions as presented here can be drawn for a flow over a flat plate in 2D. Secondly, as an alternative for the top-hat filter a Gaussian and a square cosine filter have been used, and again identical conclusions can be drawn. Concerning the third point, the results presented here are valid for two-dimensional simulations. The threedimensional case, to which future study will be directed, will lead to different results. As an example, we observed that $\tau_{i j}$ is of order $\Delta^{2}$, while it is of order $\Delta^{2 / 3}$ in the case of threedimensional, homogeneous, isotropic turbulence. ${ }^{2}$ Such differences are closely related to the fact that the shape of the energy spectrum in two dimensions is distinct from that in three dimensions. 


\section{ACKNOWLEDGEMENT}

The authors thank Luc Verhagen from Ecole Centrale de Lyon for a stimulating discussion.

\section{REFERENCES}

1. R. S. Rogallo and P. Moin, 'Numerical simulation of turbulent flows', Ann. Rev. Fluid Mech., 16, 99 (1984).

2. C. E. Leith, 'Numerical simulation of turbulent flow', in Hans Mark (Ed.), Properties of Matter Under Unusual Conditions, Interscience, 1969, p. 267.

3. D. C. Leslie and G. L. Quarini, 'The application of turbulence theory to the formulation of subgrid modelling procedures', J. Fluid Mech., 91, 65 (1979).

4. W. C. Reynolds, 'The potential and limitations of direct and large eddy simulations', in J. L. Lumley (Ed.), Whither Turbulence? Turbulence at Crossroads, Springer-Verlag, 1990, p. 313.

5. R. A. Clark, J. H. Ferziger and W. C. Reynolds, 'Evaluation of subgrid-scale models using an accurately simulated turbulent flow', J. Fluid Mech., 91, 1 (1979).

6. J. W. Deardorff, 'On the magnitude of the subgrid scale eddy viscosity coefficient', $J$. Comput. Phys., 7, 120 (1971).

7. X. Normand and M. Lesieur, 'Direct and large-eddy simulations of transition in the compressible boundary layer', Theor. Comput. Fluid Dyn., 3, 231 (1992).

8. T. A. Zang, R. B. Dahlburg and J. P. Dahlburg, 'Direct and large-eddy simulations of threedimensional compressible Navier-Stokes turbulence', Phys. Fluids A, 4, 127 (1992).

9. M. D. Love, 'Subgrid modelling studies with Burgers' equation', J. Fluid Mech., 100, 87 (1980).

10. D. Kwak, W. C. Reynolds and J. H. Ferziger, 'Three-dimensional time dependent computation of turbulent flow', Stanford Univ. Rep. TF-5, 1975.

11. C. E. Leith, 'Stochastic backscatter in a subgrid-scale model: Plane shear mixing layer', Phys. Fluids A, 2, 297 (1990).

12. M. Lesieur, C. Staquet, P. Le Roy and P. Comte, 'The mixing layer and its coherence examined from the point of view of two-dimensional turbulence', J. Fluid Mech., 192, 511 (1988).

13. W. Blumen, 'Shear layer instability of an inviscid compressible fluid', J. Fluid Mech., 40, 769 (1970).

14. N. D. Sandham and W. C. Reynolds, 'Three-dimensional simulations of large eddies in the compressible mixing layer', J. Fluid Mech., 224, 133 (1991).

15. A. W. Vreman, B. J. Geurts, J. G. M. Kuerten and P. J. Zandbergen, 'A finite volume approach to large eddy simulation of compressible, homogeneous, isotropic, decaying turbulence', Int. $j$. numer. methods fluids, 15, 799 (1992).

16. M. Lesieur, Turbulence in Fluids, Kluwer Acad. Publ., 1990.

17. U. Piomelli, T. A. Zang, G. G. Speziale and M. Y. Hussaini, 'On the large-eddy simulation of transitional wall-bounded flows', Phys. Fluids A, 2, 257 (1990). 